\title{
Phononic band gaps of elastic periodic structures: A homogenization theory study
}

\author{
Ying-Hong Liu, ${ }^{1,3, *}$ Chien C. Chang, ${ }^{1,2,3, \dagger}$ Ruey-Lin Chern, ${ }^{2,3}$ and C. Chung Chang ${ }^{1,3}$ \\ ${ }^{1}$ Division of Mechanics, Research Center for Applied Sciences, Academia Sinica, Taipei 115, Taiwan, Republic of China \\ ${ }^{2}$ Institute of Applied Mechanics, National Taiwan University, Taipei 106, Taiwan, Republic of China \\ ${ }^{3}$ Taida Institute of Mathematical Sciences, National Taiwan University, Taipei 106, Taiwan, Republic of China \\ (Received 26 April 2006; revised manuscript received 18 September 2006; published 7 February 2007)
}

\begin{abstract}
In this study, we investigate the band structures of phononic crystals with particular emphasis on the effects of the mass density ratio and of the contrast of elastic constants. The phononic crystals consist of arrays of different media embedded in a rubber or epoxy. It is shown that the density ratio rather than the contrast of elastic constants is the dominant factor that opens up phononic band gaps. The physical background of this observation is explained by applying the theory of homogenization to investigate the group velocities of the low-frequency bands at the center of symmetry $\Gamma$.
\end{abstract}

DOI: 10.1103/PhysRevB.75.054104

PACS number(s): 43.35.+d, 46.40.Cd, 63.20.-e

\section{INTRODUCTION}

Phononic crystals for elastic waves are an analog of photonic crystals for electromagnetic waves. Phononic crystals are periodic arrays of two or more elastic materials with distinct densities and elastic constants. The most distinguished feature of phononic crystals is their band gaps, and therefore phononic crystals are also called phononic bandgap materials. In the past years, we have seen steadily increasing interest in phononic crystals because of their interesting physical properties ${ }^{1-6}$ and possible engineering applications. ${ }^{7-11}$

However, there are major differences between phononic and photonic crystals that make the study of phononic crystals a separate subject from photonic crystals. First of all, dielectric materials usually support transverse electromagnetic waves, while elastic materials support both transverse as well as longitudinal elastic waves. Second, photonic materials have the largest speed of propagation in air, while elastic materials have a small (longitudinal) speed of propagation in air. Moreover, the physical properties of photonic crystals are determined by the contrast of dielectric constants, while those of phononic crystals are determined by both the contrast of elastic constants and the mass density ratio of the composed materials. In this study, we are concerned with the effects of the mass density ratio and contrast of elastic constants on major phononic band gaps and develop a theory of homogenization to examine the mechanism of the effects.

The computation of band structures is demanding, as an eigenvalue problem needs to be solved for each individual wave number in the first Brillouin zone. A fast and accurate method for computing band structures is very helpful in designing phononic band-gap materials. In the present study, we apply a method of inverse iteration with multigrid acceleration to compute the band structures of phononic crystals. This method was originally developed by the present authors to compute band structures for photonic crystals made of dielectric materials. ${ }^{12,13}$

Regarding the effects of material constants, it is natural to consider that a large contrast of elastic constants is necessary for the existence of a major band gap. This is not necessarily true as we shall show that the mass density ratio is the key factor in determining the location and size of the band gap. If the contrast of elastic constants is large, the higher-frequency bands are not very sensitive to the change of the mass density ratio, while the lower bands are heavily dependent on this change. In general, the frequency bands of the transverse modes are relatively flat compared to those of the longitudinal modes. This indicates that it is easier to open up a band gap between the bands of the transverse modes, but which is often fully blocked by the frequency bands of the longitudinal modes. As the mass density ratio is increased, the lowerfrequency bands, in particular of the longitudinal modes, shift downward in frequency and shrink significantly in size, resulting in an opening up of a major band gap. Those results could be put to a solid physical background by the theory of homogenization, which provides a good guideline for opening up band gaps. In order to indicate the underlying thought, we first develop the theory of homogenization in one dimension, then followed by the theory in two dimensions. In particular, two distinguished classes of phononic crystals are considered: media embedded in a rubber have elastic constants larger than rubbers by four or five orders in magnitude, and media embedded in an epoxy have elastic constants comparable to epoxies in magnitude.

\section{BASIC EQUATIONS}

In the present study, we consider the time-harmonic wave equation for linear, anisotropic, and elastic materials

$$
\frac{1}{\rho} \partial_{j}\left[C_{i j m n} \partial_{n} u_{m}(\mathbf{r})\right]+\omega^{2} u_{i}(\mathbf{r})=0,
$$

where $u_{i}(i=1,2,3)$ are the displacements, and $\rho=\rho(\mathbf{r})$ and $C_{i j m n}=C_{i j m n}(\mathbf{r})$ are the mass density and elastic stiffness tensor, respectively. For a two-dimensional problem in the $x y$ plane with out-of-plane propagation in the $z$ direction, Eq. (1) can be written for cubic crystals as

$$
-\frac{1}{\rho}\left[\begin{array}{lll}
L_{x x} & L_{x y} & L_{x z} \\
L_{y x} & L_{y y} & L_{y z} \\
L_{z x} & L_{z y} & L_{z z}
\end{array}\right]\left[\begin{array}{l}
u_{x} \\
u_{y} \\
u_{z}
\end{array}\right]=\omega^{2}\left[\begin{array}{l}
u_{x} \\
u_{y} \\
u_{z}
\end{array}\right],
$$

where $L_{i j}(i, j=1,2,3)$ are detailed in the Appendix. For periodic structures, it is sufficient to solve the problem in one 


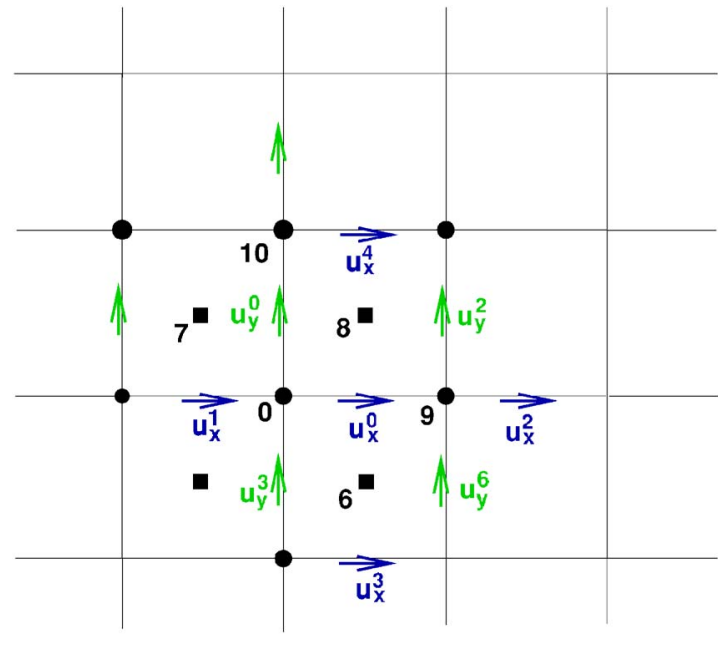

FIG. 1. (Color online) The stagger mesh is widely applied with a finite-difference time domain algorithm. First, we split the components of the displacement onto different mesh points-i.e., moving forward the $x$ component of the displacement half of mesh length in the $x$ direction-and treat the same way for the $y$ component of the displacement in the $y$ direction. Second, we put the physical properties of the material in different mesh points. For example, in the cubic system, the mass densities $C_{11}$ and $C_{12}$ are assigned to circle mesh points and $C_{44}$ is assigned to rectangular points.

unit cell, along with the Bloch condition satisfied at the cell boundary,

$$
u_{j}\left(\mathbf{r}+\mathbf{a}_{i}\right)=e^{i \mathbf{k} \cdot \mathbf{a}_{i}} u_{j}(\mathbf{r}),
$$

where $\mathbf{k}$ is the wave vector and $\mathbf{a}_{i}(i=1,2)$ are the lattice translation vectors. A central finite-difference scheme ${ }^{14}$ is used to discretize Eq. (2). The positions of $u_{x}$ and $u_{y}$ are offset by a half grid size in their own directions, respectively, as shown in Fig. 1. There are two points we want to mention. First, we separate the components of the displacement half mesh in its own direction. Second, the elastic constants and the mass density are arranged into different areas. In our study, the mass density and elastic constants $C_{11}$ and $C_{12}$ are assigned to the mesh points but $C_{44}$ is assigned to the center of the mesh zone, as shown in the figure. This arrangement is helpful for the convergence of numerical results. Then we obtain the discretized eigenvalue problem

$$
\mathbf{A u}=\lambda \mathbf{u},
$$

where we have applied condition (3). The eigensystem is solved by the method of inverse iteration with multigrid acceleration as mentioned in the Introduction.

\section{RESULTS AND DISCUSSION}

Let us consider a square lattice of square cylinders of materials embedded in a rubber. The physical constants of the embedded materials are listed in Table I (Refs. 15 and 16); all the embedded materials have elastic constants larger than the rubber by four or five orders in magnitude.

First, we consider the band structure of the C/rubber system with the filling fraction $f=0.36$ (Fig. 2). Although the
TABLE I. The unit of the mass density is $\mathrm{g} / \mathrm{cm}^{3}$ and the elastic constants are in $10^{9} \mathrm{~N} / \mathrm{m}^{2}$. These physical constants are used for investigating two-dimensional phononic crystals.

\begin{tabular}{lccc}
\hline \hline Medium & $\rho$ & $C_{11}$ & $C_{44}$ \\
\hline Ice & 0.94 & 13.79 & 3.18 \\
$\mathrm{C}$ & 1.75 & 310 & 88.5 \\
$\mathrm{AlAs}$ & 3.76 & 120.2 & 58.9 \\
$\mathrm{GaAs}$ & 5.36 & 118.8 & 59.4 \\
$\mathrm{Ni}$ & 8.97 & 311.61 & 92.93 \\
$\mathrm{Ag}$ & 10.64 & 152.68 & 40.44 \\
$\mathrm{~Pb}$ & 11.6 & 72.1 & 14.9 \\
$\mathrm{~W}$ & 19.3 & 500.03 & 151.31 \\
Rubber & 1.3 & $6.8 \times 10^{-4}$ & $4.0 \times 10^{-5}$ \\
Epoxy & 1.20 & 9.61 & 1.61 \\
\hline \hline
\end{tabular}

contrast of the elastic constant between carbon and rubber is quite large, no full band gaps are observed in this structure (Fig. 3). In fact, a band gap exists between the first few transverse modes of shear horizontal $\left(\mathrm{SH}_{1}\right)$ and shear vertical $\left(\mathrm{SV}_{1}\right)$ branches. However, this band gap is blocked by the first longitudinal mode of pressure $(L)$ branch. If carbon cylinders are replaced by heavier Pb cylinders, ${ }^{16}$ then large full band gaps can be opened up. Figure 4 shows the band structures of $\mathrm{Pb}$ cylinders embedded in a rubber background for the same filling fraction. In this case, there is a large contrast in the mass density between the embedded material and the background. The frequencies of the first $L$, the first SV, and the first two $\mathrm{SH}$ branches are significantly reduced to lower values, while the frequency of the second SV branches remains little changed and becomes flattened. It is also observed that the higher-frequency bands are much less sensitive to the change of the physical constants of the embedded material. As a result, two large full band gaps, denoted by $\mathrm{Ba}$ and $\mathrm{Bb}$, respectively, are opened up and separated by a nearly straight band. The question is why the difference in the mass density ratio is more effective in opening up a band gap than a large contrast of elastic constants. We now attempt to answer this question by developing a theory of homogenization.

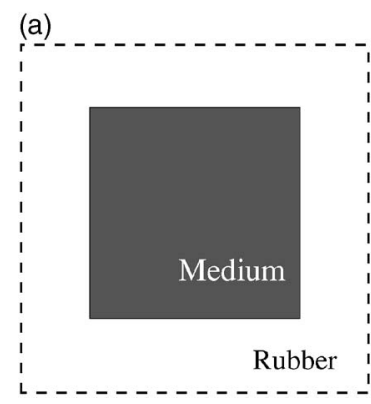

(b)
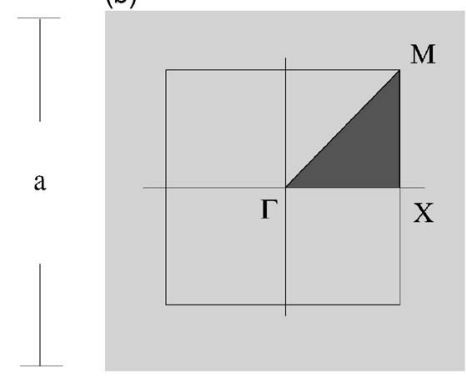

FIG. 2. (a) The medium/rubber square lattice. The filling fraction is 0.36. The first simulated case is the C/rubber system and the second is the Pb/rubber system. (b) The first Brillouin zone and the special points of symmetry. 


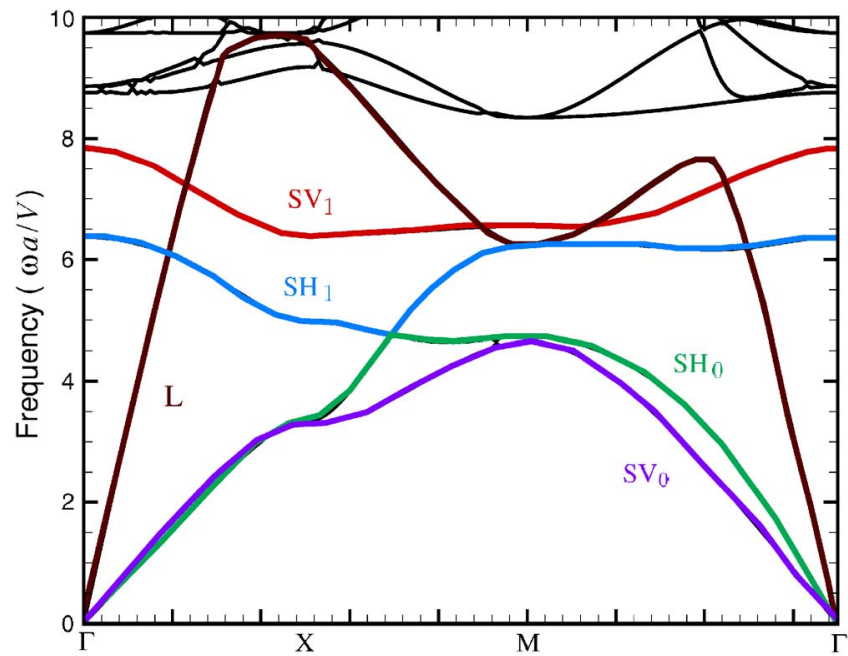

FIG. 3. (Color online) The band structure of the array of carbon squares embedded in a rubber background. The filling fraction $f$ of the carbon is 0.36 . The frequency is normalized by $V / a$ where $V$ is the transverse velocity of the rubber.

\section{A. One-dimensional homogenization}

In order to open up a band gap, one possibility is to lower the first few frequency bands. It is plausible that if we can reduce the slopes of the low-frequency bands at the point $\Gamma$, then these bands may entirely shift downward. The slopes are actually the group velocities. The group velocity of the composite material at the low-frequency limit for periodic structures can be determined by applying the theory of homogenization (see, e.g., Ref. 17). For this purpose, we consider the model problem of one-dimensional elasticity:

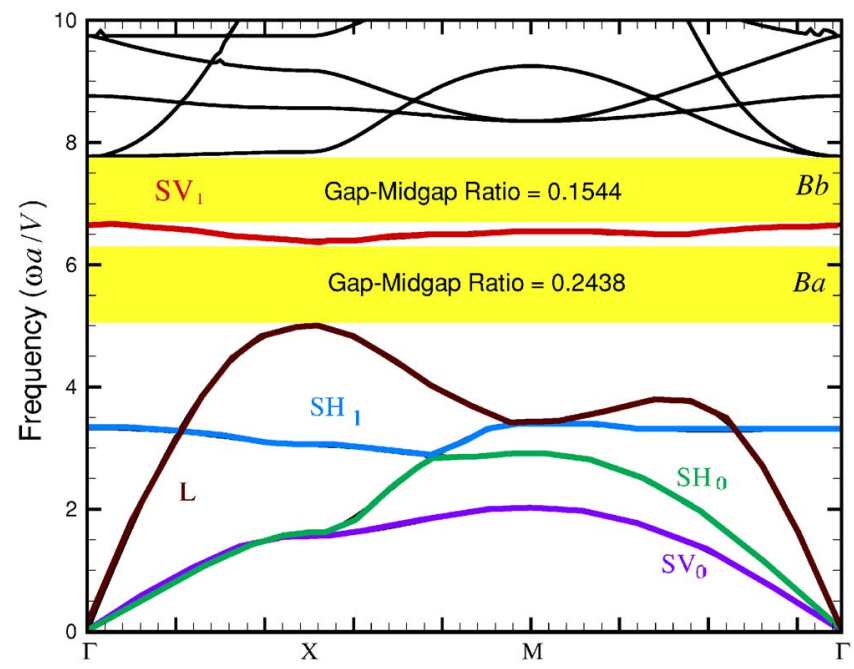

FIG. 4. (Color online) The band structure of an array of $\mathrm{Pb}$ squares embedded in a rubber background. The filling fraction $f$ of $\mathrm{Pb}$ is 0.36 . The frequency is normalized by $V / a$ where $V$ is the transverse velocity of rubber. $\mathrm{Ba}$ and $\mathrm{Bb}$ denote first and second band gaps.

$$
\frac{\partial}{\partial x}\left(E \frac{\partial}{\partial x} u\right)=\rho \frac{\partial^{2} u}{\partial t^{2}},
$$

where $\rho$ denotes the mass density and $E$ is Young's modulus, both of which vary with a period $a$ of which material 1 occupies a proportion $f a$, while material 2 occupies $(1-f) a$.

Let us consider a wave propagating with a large wavelength $l$-i.e., $\epsilon=a / l \ll 1$. It is convenient to do nondimensional analysis by scaling; we introduce $x \rightarrow a x, E \rightarrow E_{c} E, \rho$ $\rightarrow \rho_{c} \rho$, and $t \rightarrow 2 \pi t / \omega_{c}$ where $E_{c}, \rho_{c}$, and $\omega_{c}$ are characteristic values of Young's modulus, mass density, and frequency. Then we obtain

$$
\frac{\partial}{\partial x}\left(E \frac{\partial}{\partial x} u\right)=\frac{a^{2}}{4 \pi^{2} E_{c} /\left(\rho_{c} \omega_{c}^{2}\right)} \rho \frac{\partial^{2} u}{\partial t^{2}},
$$

where we identify $l^{2}=4 \pi^{2} E_{c} /\left(\rho_{c} \omega_{c}^{2}\right)$, and the scaled Eq. (5) becomes

$$
\frac{\partial}{\partial x}\left(E \frac{\partial}{\partial x} u\right)=\epsilon^{2} \rho \frac{\partial^{2} u}{\partial t^{2}}
$$

Now we introduce two scales, $x=x$ (fine scale) and $x^{\prime}=\epsilon x$ (coarse-grained), for further analysis. The displacement is considered as a function of $x$ and $x^{\prime}-$ i.e., $u=u\left(x, x^{\prime}\right)$; then,

$$
\frac{\partial}{\partial x} \rightarrow \frac{\partial}{\partial x}+\epsilon \frac{\partial}{\partial x^{\prime}}
$$

Expanding $u$ in powers of $\epsilon$,

$$
u=u^{0}+\epsilon u^{1}+\epsilon^{2} u^{2}+\cdots,
$$

we obtain

$$
\begin{aligned}
& \left(\frac{\partial}{\partial x}+\epsilon \frac{\partial}{\partial x^{\prime}}\right)\left[E\left(\frac{\partial}{\partial x}+\epsilon \frac{\partial}{\partial x^{\prime}}\right)\left(u^{0}+\epsilon u^{1}+\epsilon^{2} u^{2}+\cdots\right)\right] \\
& =\epsilon^{2} \rho \frac{\partial^{2} u^{0}}{\partial t^{2}}+\cdots
\end{aligned}
$$

Next we collect terms of the same power in $\epsilon$. For the order $O\left(\epsilon^{0}\right)$, we get

$$
\frac{\partial}{\partial x}\left(E \frac{\partial u^{0}}{\partial x}\right)=0
$$

where $u^{0}$ denotes the coarse-grained displacement, and it is evident that it depends on $x^{\prime}$ only. For the order $O\left(\epsilon^{1}\right)$, we obtain

$$
\frac{\partial}{\partial x}\left[E\left(\frac{\partial u^{1}}{\partial x}+\frac{\partial u^{0}}{\partial x^{\prime}}\right)\right]=0 .
$$

A general solution of $u^{1}$ is given by

$$
u^{1}=Q\left(x, x^{\prime}\right) \frac{\partial u^{0}}{\partial x^{\prime}}+\bar{u}^{1}\left(x^{\prime}\right),
$$

where $Q\left(x, x^{\prime}\right)$ is a periodic function in $x$ of period $a$ and $\bar{u}^{1}\left(x^{\prime}\right)$ is independent of $x$. Substituting $u^{1}$ of Eq. (13) into Eq. (12), we obtain 
TABLE II. Group velocities of the lowest-frequency bands at the point $\Gamma$ for a one-dimensional crystal. The unit of velocity is $\mathrm{km} / \mathrm{s} . V_{n, 1 D}^{L}$ and $V_{n, 1 D}^{S}$ are the velocities of longitudinal waves and shear waves, respectively, obtained from the numerical results. $V_{h, 1 D}^{L}$ and $V_{h, 1 D}^{S}$ are obtained from the theory of one-dimensional homogenization.

\begin{tabular}{lcccc}
\hline \hline Composite & $V_{n, 1 D}^{L}$ & $V_{n, 1 D}^{S}$ & $V_{h, 1 D}^{L}$ & $V_{h, 1 D}^{S}$ \\
\hline C/rubber & 0.0271 & 0.00655 & 0.0270 & 0.0065 \\
Pb/rubber & 0.0145 & 0.0035 & 0.0145 & 0.0035 \\
\hline \hline
\end{tabular}

$$
\frac{\partial}{\partial x}\left[E\left(1+\frac{\partial Q}{\partial x}\right)\right]=0
$$

A simple integration gives

$$
Q=-x+D_{1} \int_{x_{0}}^{x_{0}+x} \frac{d \tilde{x}}{E}+D_{2},
$$

where $D_{1}$ and $D_{2}$ are functions of $x^{\prime}$ only and $x_{0}$ is a reference point. Since $Q$ must be a periodic function of $x$ with period $a, D_{1}$ needs to satisfy

$$
D_{1}=\left(\frac{1}{a} \int_{x_{0}}^{x_{0}+a} \frac{d \tilde{x}}{E}\right)^{-1}
$$

and $D_{2}$ can simply be taken to be zero. The results suggest that we define the effective Young's modulus

$$
E_{e}=D_{1}=\left\langle\frac{1}{E}\right\rangle^{-1}=\frac{E_{1} E_{2}}{f E_{2}+(1-f) E_{1}},
$$

where $E_{1}$ and $E_{2}$ are Young's moduli of embedded and host materials, respectively. Finally, gathering terms for the $O\left(\epsilon^{2}\right)$, we have

$$
\frac{\partial}{\partial x^{\prime}}\left[E\left(\frac{\partial u^{1}}{\partial x}+\frac{\partial u^{0}}{\partial x^{\prime}}\right)\right]+\frac{\partial}{\partial x}\left[E\left(\frac{\partial u^{2}}{\partial x}+\frac{\partial u^{1}}{\partial x^{\prime}}\right)\right]=\rho \frac{\partial^{2} u^{0}}{\partial t^{2}}
$$

In order to see the behavior on a macroscale, we average Eq. (18) in the unit cell. The second term after being averaged with respect to $x$ becomes zero because of the periodic boundary conditions. Then the resulting averaged equation with using Eqs. (13), (15), and (17) becomes

$$
\frac{\partial}{\partial x^{\prime}}\left(E_{e} \frac{\partial u^{0}}{\partial x^{\prime}}\right)=\langle\rho\rangle \frac{\partial^{2} u^{0}}{\partial t^{2}}
$$

where $\langle\rho\rangle$ is the mean mass density,

$$
\langle\rho\rangle=f \rho_{1}+(1-f) \rho_{2} .
$$

Equation (19) only depends on $x^{\prime}$ and describes the macroscale elastic waves propagating along the composite material under the long-wave approximation. This explains why $E_{e}$ is called effective Young's modulus. The effective velocity is given by
TABLE III. Group velocities of the lowest-frequency bands at the point $\Gamma$ for a two-dimensional crystal. The unit of velocity is $\mathrm{km} / \mathrm{s} . V_{n, 2 D}^{L}$ and $V_{n, 2 D}^{S V}$ are the velocities of longitudinal waves and shear vertical waves, respectively, obtained from the numerical results. $V_{h, 1 D}^{L}$ and $V_{h, 1 D}^{S}$ are obtained from the theory of onedimensional homogenization.

\begin{tabular}{lcccc}
\hline \hline Composite & $V_{n, 2 D}^{L}$ & $V_{n, 2 D}^{S V}$ & $V_{h, 1 D}^{L}$ & $V_{h, 1 D}^{S}$ \\
\hline C/rubber & 0.0289 & 0.0078 & 0.0270 & 0.0065 \\
Pb/rubber & 0.0154 & 0.0042 & 0.0145 & 0.0035 \\
\hline \hline
\end{tabular}

$$
v_{e}=\sqrt{\frac{E_{e}}{\langle\rho\rangle}}=\sqrt{\frac{E_{1} E_{2}}{f E_{2}+(1-f) E_{1}} \frac{1}{f \rho_{1}+(1-f) \rho_{2}}} .
$$

The results (17) and (20) indicate that the effective Young's modulus $E_{e}$ is mainly determined by the less rigid material, $E_{e}=E_{2} /(1-f)$ if $E_{1} \gg E_{2}$, while the mean density is determined by the heavier material, $\langle\rho\rangle=f \rho_{1}$ if $\rho_{1} \gg \rho_{2}$. In our simulation, $E_{1}$ and $E_{2}$ stand for the elastic constants of the embedded materials and the host (rubber), respectively, with $E_{1} \gg E_{2}$. Therefore,

$$
v_{e} \approx \sqrt{\frac{E_{2}}{1-f f \rho_{1}+(1-f) \rho_{2}}} .
$$

The simple theory is particularly accurate for onedimensional crystal (Table II), and is less satisfactory for two-dimensional crystals (Table III). In both $\mathrm{C} /$ rubber and $\mathrm{Pb} /$ rubber systems, $\mathrm{C}$ and $\mathrm{Pb}$ have a much larger Young's modulus than rubber. Thus both composite systems have the same effect by increasing the Young's modulus from rubber's $E_{2}$ to $E_{2} /(1-f)=E_{2} / 0.64$. The effective group velocity of the two composite systems, differing from that of rubber, is mainly determined by the difference in density between the host and embedded materials.

For the longitudinal waves, we have

$$
\begin{gathered}
v_{e, \text { long }}^{\mathrm{C} / r u b}=\sqrt{\frac{C_{11}}{1-f f \rho_{1}+(1-f) \rho_{2}}} \approx 1.26 v_{\text {long }}^{\text {rub }}, \\
v_{e, \text { long }}^{\mathrm{Pb} / \text { ub }}=\sqrt{\frac{C_{11}}{1-f f \rho_{1}+(1-f) \rho_{2}}} \approx 0.672 v_{\text {long }}^{\text {rub }} .
\end{gathered}
$$

The same results apply to the effective transverse velocity by simply replacing $C_{11}$ with $C_{44}$. The results (23) and (24) explain why the $\mathrm{Pb}$ /rubber systems compared to the $\mathrm{C} /$ rubber system lower the first few bands very effectively, especially in longitudinal modes, which covers some band gaps produced by shear modes, thus opening up a band gap. On the other hand, we may embed a less rigid material in rubber (but with comparable density) to reduce the group velocity, at the point $\Gamma$ and thus lower the lower-frequency bands.

To see a closer comparison between numerical and theoretical results, we elaborate below on the theory of twodimensional homogenization. 


\section{B. Two-dimensional homogenization}

Let us start with the time-dependent form of the wave equation

$$
\frac{\partial}{\partial x_{j}}\left[C_{i j m n} \frac{\partial u_{m}}{\partial x_{n}}\right]=\rho \frac{\partial^{2} u_{i}}{\partial t^{2}} .
$$

The same procedure as the one-dimensional problem leads to

$$
\begin{aligned}
& \left(\frac{\partial}{\partial x_{j}}+\epsilon \frac{\partial}{\partial x_{j}^{\prime}}\right)\left[C_{i j m n}\left(\frac{\partial}{\partial x_{n}}+\epsilon \frac{\partial}{\partial x_{n}^{\prime}}\right)\left(u_{m}^{0}+\epsilon u_{m}^{1}+\epsilon^{2} u_{m}^{2}+\cdots\right)\right] \\
& =\epsilon^{2} \rho \frac{\partial^{2} u_{i}^{0}}{\partial t^{2}}+\cdots
\end{aligned}
$$

where $i, m=1,2,3$ and $j, n=1,2$. Then we collect terms in different powers of $\epsilon$. At the order $O\left(\epsilon^{0}\right)$, we have

$$
\frac{\partial}{\partial x_{j}}\left(C_{i j m n} \frac{\partial u_{m}^{0}}{\partial x_{n}}\right)=0 \text {. }
$$

From the discussion of the previous suggestion, we know that $u_{m}^{0}=u_{m}^{0}\left(\mathbf{x}^{\prime}\right)$ depends on $\mathbf{x}^{\prime}$ only. At the order $O\left(\epsilon^{1}\right)$, we get

$$
\frac{\partial}{\partial x_{j}}\left[C_{i j m n}\left(\frac{\partial u_{m}^{0}}{\partial x_{n}^{\prime}}+\frac{\partial u_{m}^{1}}{\partial x_{n}}\right)\right]=0 .
$$

The key step is to solve Eq. (28) for $u_{m}^{1}$. In analog with Eq. (13), we assume the form of solution

$$
u_{m}^{1}=b_{k}^{(m)} \frac{\partial u_{m}^{0}}{\partial x_{k}^{\prime}}+\bar{u}_{m}^{1}\left(\mathbf{x}^{\prime}\right),
$$

where $\bar{u}_{m}^{1}\left(\mathbf{x}^{\prime}\right)$ is independent of $\mathbf{x}$ and $b_{k}^{(m)}$ has no summation in the index $m$. The solution form connects the perturbed solutions at the zeroth order and the first order. Substituting $u_{m}^{1}$ of Eq. (29) into Eq. (28), we obtain

$$
\frac{\partial}{\partial x_{j}}\left[C_{i j m n}\left(\delta_{n k}+\frac{\partial b_{k}^{(m)}}{\partial x_{n}}\right)\right] \frac{\partial u_{m}^{0}}{\partial x_{k}^{\prime}}=0 .
$$

At the order $O\left(\epsilon^{2}\right)$, we have

$$
\begin{aligned}
& \frac{\partial}{\partial x_{j}^{\prime}}\left[C_{i j m n}\left(\frac{\partial u_{m}^{0}}{\partial x_{n}^{\prime}}+\frac{\partial u_{m}^{1}}{\partial x_{n}}\right)\right]+\frac{\partial}{\partial x_{j}}\left[C_{i j m n}\left(\frac{\partial u_{m}^{1}}{\partial x_{n}^{\prime}}+\frac{\partial u_{m}^{2}}{\partial x_{n}}\right)\right] \\
& \quad=\rho \frac{\partial^{2} u_{i}^{0}}{\partial t^{2}} .
\end{aligned}
$$

Now Eq. (31) is averaged with respect to $\mathbf{x}$ over the unit cell. The second term is immediately averaged to give zero because of periodic boundary conditions. Then we have

$$
\frac{\partial}{\partial x_{j}^{\prime}}\left\langle C_{i j m n}\left(\delta_{n k}+\frac{\partial b_{k}^{(m)}}{\partial x_{n}}\right)\right\rangle \frac{\partial u_{m}^{0}}{\partial x_{k}^{\prime}}=\langle\rho\rangle \frac{\partial^{2} u_{i}^{0}}{\partial t^{2}} .
$$

In analog with Eq. (25), this motivates us to define the effective elastic constants as

$$
C_{i j m k}^{e}=\left\langle C_{i j m n}\left(\delta_{n k}+\frac{\partial b_{k}^{(m)}}{\partial x_{n}}\right)\right\rangle,
$$

and then Eq. (32) becomes

$$
\frac{\partial}{\partial x_{j}^{\prime}} C_{i j m k}^{e} \frac{\partial u_{m}^{0}}{\partial x_{k}^{\prime}}=\langle\rho\rangle \frac{\partial^{2} u_{i}^{0}}{\partial t^{2}} .
$$

In contrast to the one-dimensional problem, we do not have a closed-form formula of effective elastic constants. Instead, they have to be obtained by solving

$$
\frac{\partial}{\partial x_{j}}\left[C_{i j m n}\left(\delta_{n k}+\frac{\partial b_{k}^{(m)}}{\partial x_{n}}\right)\right]=0,
$$

as indicated by Eq. (30), where $\partial u_{m}^{0} / \partial x_{k}^{\prime}$ could be general functions of $x_{k}^{\prime}$. It is straightforward to see that Eq. (35) reduces to Eq. (14) if we consider the one-dimensional elastic wave equation (5).

Equation (35) is the key to the two-dimensional homogenization. What we need for the effective $C_{i j m k}^{e}$ are $C_{i j m n}$ and $b_{k}^{(m)}$ as shown in Eq. (33). This equation also indicates that the determination of $b_{k}^{(m)}$ depends on the spatial property of $C_{i j m n}$ and thus the possibility of improvement of twodimensional homogenization over the one-dimensional theory. Moreover, the above formulation is valid for general linear elastic materials in both two and three dimensions.

Now we consider the two-dimensional elastic wave equations of the cubic materials. For shear vertical (SV) modes, we have

$$
L_{z z} u_{z}=\rho \frac{\partial^{2} u_{z}}{\partial t^{2}}
$$

and for longitudinal-shear horizontal $(L-\mathrm{SH})$ modes, we have

$$
\begin{aligned}
& L_{x x} u_{x}+L_{x y} u_{y}=\rho \frac{\partial^{2} u_{x}}{\partial t^{2}}, \\
& L_{y x} u_{x}+L_{y y} u_{y}=\rho \frac{\partial^{2} u_{y}}{\partial t^{2}} .
\end{aligned}
$$

SV modes. Consider the SV mode. The coarse-grained equation is given by

$$
\frac{\partial}{\partial x_{i}^{\prime}}\left(\left\langle C_{44}^{i j}\right\rangle \frac{\partial u_{z}^{0}}{\partial x_{j}^{\prime}}\right)=\langle\rho\rangle \frac{\partial^{2} u_{z}^{0}}{\partial t^{2}},
$$

where $i, j=1,2$ and $u_{z}^{0}$ denotes the coarse-grained displacement of the $z$ component, and

$$
\begin{gathered}
\langle\rho\rangle=\frac{1}{\Omega} \int_{\Omega} \rho d \Omega, \\
\left\langle C_{44}^{i j}\right\rangle=\frac{1}{\Omega} \int_{\Omega} C_{44}\left(\delta_{i j}+\frac{\partial b_{j}}{\partial x_{i}}\right) d \Omega .
\end{gathered}
$$

Here, $\Omega$ is the domain of the unit cell, $C_{44}=C_{1313}$ or $C_{2323}$ and $b_{j}$ satisfying

$$
\frac{\partial}{\partial x_{i}}\left[C_{44}\left(\delta_{i j}+\frac{\partial b_{j}}{\partial x_{i}}\right)\right]=0,
$$

and must, in general, be solved numerically. Since Eq. (42) is supplement by the periodic boundary conditions, we append the additional condition $\left\langle b_{j}\right\rangle=0$ to solve Eq. (42) uniquely. 
This effective shear vertical group velocity is given by

$$
V_{e, 2 D}^{S V}=\sqrt{\frac{\left\langle C_{44}^{11}\right\rangle}{\langle\rho\rangle}} .
$$

L-SH Modes. Consider the wave equations of the mixed mode. The coarse-grained equation is given by

$$
\begin{gathered}
\frac{\partial}{\partial x^{\prime}}\left(\left\langle C_{11}\right\rangle \frac{\partial u_{x}^{0}}{\partial x^{\prime}}\right)+\frac{\partial}{\partial y^{\prime}}\left(\left\langle C_{44}\right\rangle \frac{\partial u_{x}^{0}}{\partial y^{\prime}}\right)+\frac{\partial}{\partial x^{\prime}}\left(\left\langle C_{12}\right\rangle \frac{\partial u_{y}^{0}}{\partial y^{\prime}}\right) \\
+\frac{\partial}{\partial y^{\prime}}\left(\left\langle C_{44}^{\prime}\right\rangle \frac{\partial u_{y}^{0}}{\partial x^{\prime}}\right)+\operatorname{Res}=\langle\rho\rangle \frac{\partial^{2} u_{x}^{0}}{\partial t^{2}} .
\end{gathered}
$$

The effective elastic constants are given by

$$
\begin{aligned}
& \left\langle C_{11}\right\rangle=\frac{1}{\Omega} \int_{\Omega} C_{11}\left(1+\frac{\partial b_{1}^{(1)}}{\partial x}\right) d \Omega, \\
& \left\langle C_{44}\right\rangle=\frac{1}{\Omega} \int_{\Omega} C_{44}\left(1+\frac{\partial b_{2}^{(1)}}{\partial y}\right) d \Omega, \\
& \left\langle C_{12}\right\rangle=\frac{1}{\Omega} \int_{\Omega} C_{12}\left(1+\frac{\partial b_{2}^{(2)}}{\partial y}\right) d \Omega, \\
& \left\langle C_{44}^{\prime}\right\rangle=\frac{1}{\Omega} \int_{\Omega} C_{44}\left(1+\frac{\partial b_{1}^{(2)}}{\partial x}\right) d \Omega,
\end{aligned}
$$

where $C_{11}=C_{1111}$ or $C_{2222}, C_{44}=C_{1212}$ and $C_{12}=C_{1122}$, and Res denotes the residual,

$$
\begin{aligned}
\operatorname{Res}= & \frac{1}{\Omega} \int_{\Omega} \frac{\partial}{\partial y^{\prime}}\left[C_{44} \frac{\partial b_{1}^{(1)}}{\partial y} \frac{\partial u_{x}^{0}}{\partial x^{\prime}}+C_{44} \frac{\partial b_{2}^{(2)}}{\partial x} \frac{\partial u_{y}^{0}}{\partial y^{\prime}}\right] \\
& +\frac{\partial}{\partial x^{\prime}}\left[C_{11} \frac{\partial b_{2}^{(1)}}{\partial x} \frac{\partial u_{x}^{0}}{\partial y^{\prime}}+C_{12} \frac{\partial b_{1}^{(2)}}{\partial y} \frac{\partial u_{y}^{0}}{\partial x^{\prime}}\right] d \Omega,
\end{aligned}
$$

where $b_{i}^{(m)}$ are functions satisfying

$$
\begin{aligned}
& \frac{\partial}{\partial x}\left[C_{11} \frac{\partial b_{1}^{(1)}}{\partial x}\right]+\frac{\partial}{\partial y}\left[C_{44} \frac{\partial b_{1}^{(1)}}{\partial y}\right]=-\frac{\partial C_{11}}{\partial x}, \\
& \frac{\partial}{\partial x}\left[C_{11} \frac{\partial b_{2}^{(1)}}{\partial x}\right]+\frac{\partial}{\partial y}\left[C_{44} \frac{\partial b_{2}^{(1)}}{\partial y}\right]=-\frac{\partial C_{44}}{\partial y}, \\
& \frac{\partial}{\partial x}\left[C_{12} \frac{\partial b_{1}^{(2)}}{\partial y}\right]+\frac{\partial}{\partial y}\left[C_{44} \frac{\partial b_{1}^{(2)}}{\partial x}\right]=-\frac{\partial C_{44}}{\partial y}, \\
& \frac{\partial}{\partial x}\left[C_{12} \frac{\partial b_{2}^{(2)}}{\partial y}\right]+\frac{\partial}{\partial y}\left[C_{44} \frac{\partial b_{2}^{(2)}}{\partial x}\right]=-\frac{\partial C_{12}}{\partial x} .
\end{aligned}
$$

The effective shear horizontal and longitudinal group velocities are given, respectively, by

$$
V_{e, 2 D}^{S H}=\sqrt{\frac{\left\langle C_{44}\right\rangle}{\langle\rho\rangle}}, \quad V_{e, 2 D}^{L}=\sqrt{\frac{\left\langle C_{11}\right\rangle}{\langle\rho\rangle}} .
$$

Table IV shows the comparison of group velocity at point $\Gamma$ between the numerical results and those predicted by two-
TABLE IV. Group velocities of the lowest-frequency bands at the point $\Gamma$ for a two-dimensional crystal. The unit of velocity is $\mathrm{km} / \mathrm{s} . V_{n, 2 D}^{L}, V_{n, 2 D}^{S H}$, and $V_{n, 2 D}^{S V}$ are the velocities of longitudinal waves, shear horizontal, and vertical waves, respectively, obtained from the numerical results. $V_{h, 2 D}^{L}, V_{h, 2 D}^{S H}$, and $V_{h, 2 D}^{S V}$ are obtained from the theory of two-dimensional homogenization.

\begin{tabular}{lcccccc}
\hline \hline Composite & $V_{n, 2 D}^{L}$ & $V_{n, 2 D}^{S H}$ & $V_{n, 2 D}^{S V}$ & $V_{h, 2 D}^{L}$ & $V_{h, 2 D}^{S H}$ & $V_{h, 2 D}^{S V}$ \\
\hline C/rubber & 0.0289 & 0.0072 & 0.0078 & 0.0294 & 0.0078 & 0.0078 \\
Pb/rubber & 0.0154 & 0.0040 & 0.0042 & 0.0159 & 0.0042 & 0.0042 \\
\hline
\end{tabular}

dimensional homogenization. The results show significant improvement over the comparison listed in Table III between two-dimensional numerical results and the results of onedimensional homogenization.

The one-dimensional homogenization indicates that a large contrast of mass density is necessary for producing full band gaps in phononic crystals, no matter how large the contrast of elastic constants is. In order to illustrate this general trend for two-dimensional systems, we simulate different materials (listed in Table I with given physical constants) embedded in rubber. In Fig. 5, we plot the band-gap ratios of $\mathrm{Ba}$ and $\mathrm{Bb}$ (defined in Fig. 4) for different materials embedded in a rubber background. For ice, carbon, and aluminum, with comparable mass densities of that of rubber, neither band gap $\mathrm{Ba}$ nor $\mathrm{Bb}$ is observed. The band gaps are found easily in heavier $\mathrm{Ni}, \mathrm{Ag}$, and $\mathrm{Pb} /$ rubber systems. As the mass density is increased above that of GaAs, band gap Ba opens up and its size (band-gap-midgap ratio) increases linearly up

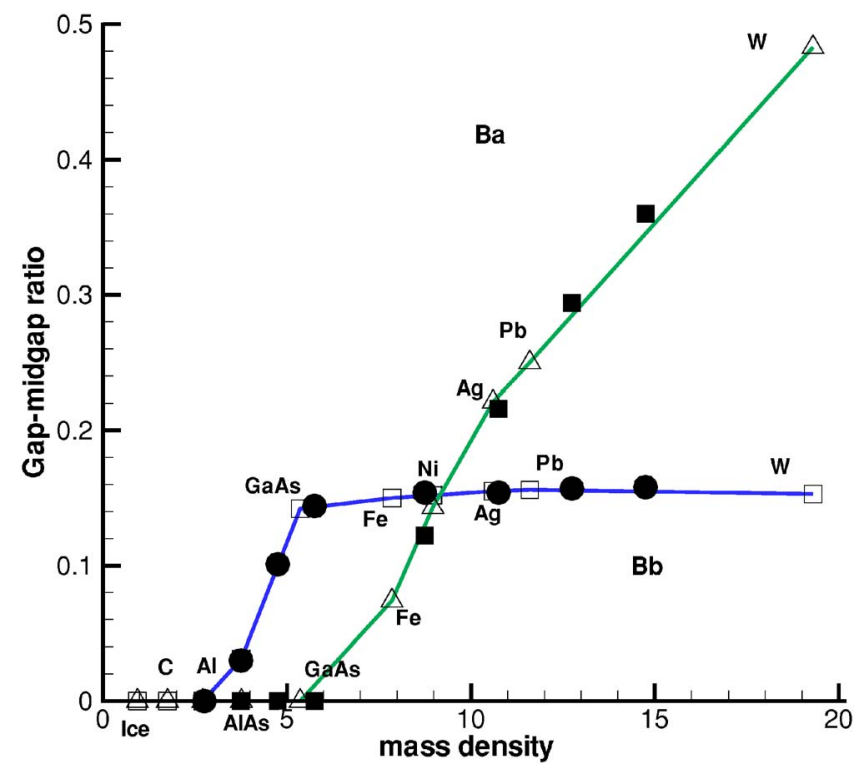

FIG. 5. (Color online) The band-gap size for different medium/ rubber systems. The unit of mass density is $\mathrm{g} / \mathrm{cm}^{3}$. The mass density of rubber is 1.3 , lying between carbon and ice. The open triangle symbols and open square symbols denote the gap-midgap ratios of $\mathrm{Ba}$ and $\mathrm{Bb}$. Here, the solid symbols denote artificial embedded materials which have the same elastic constants of carbon, but with different mass densities. All the cases have fixed filling fraction $f=0.36$ for the embedded medium. 


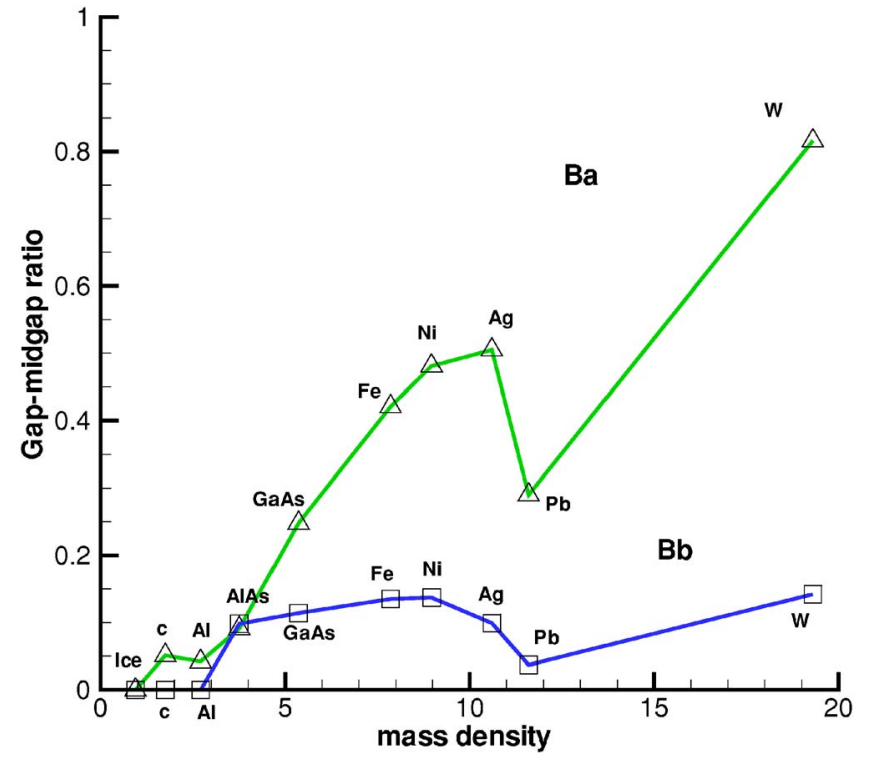

FIG. 6. (Color online) The band-gap size for different medium/ epoxy systems. The unit of mass density is $\mathrm{g} / \mathrm{cm}^{3}$. The mass density of epoxy is 1.2 , lying between carbon and ice. The open triangle symbols and open square symbols denote the gap-midgap ratios of $\mathrm{Ba}$ and $\mathrm{Bb}$. All the cases have fixed filling fraction $f$ $=0.36$ for the embedded medium.

to $W$. On the other hand, band gap Bb opens up as the density ratio is increased above that of $\mathrm{Al}$ and increases linearly to GaAs, where we see a saturated size 0.15 . It is also interesting to see if the host material rubber is replaced by a more rigid material like epoxy. Epoxy has $Q$ density about that of rubber but has elastic constants comparable to other materials in magnitude. Figure 6 shows the results for comparison. The general trend of the two bands $\mathrm{Ba}$ and $\mathrm{Bb}$ for medium/ epoxy systems is not too much different from the medium/ rubber systems though the comparable elastic constants of the medium and host complicated their effects of homogenization.

\section{CONCLUDING REMARKS}

In the present study, we have applied a fast algorithminverse iteration with multigrid acceleration-to compute the band structures of phononic crystals. A critical issue is addressed as how to open up a large band gap for phononic crystals. It is shown, by the theory of homogenization in one as well as two dimensions, how the mass density ratio and the contrast of elastic constants affect the size of major phononic band gaps. In particular, it is quite efficient to open up a band gap by lowering the group velocities of the lowfrequency bands at the center $\Gamma$. One-dimensional homogenization shows that the effective mass density is the areaaveraged density of the host and embedded materials, while this is true for the elastic constants if we consider their inverses. In contrast, two-dimensional homogenization does not exhibit this simple average for the elastic constants. Instead, before averaging we have to solve a system of equations that take care of the detailed spatial properties of the elastic constants. This explains why the two-dimensional homogenization shows significantly improved results over the one-dimensional theory in predicting the group velocities of the lowest-frequency bands at the center of symmetry $\Gamma$. The current method of analysis is applied to three-dimensional problems; the results will be reported elsewhere.

\section{ACKNOWLEDGMENTS}

The work is supported in part by the National Science Council of the Republic of China (Taiwan) under Contract No. NSC 94-2212-E-002-047 and the Ministry of Economic Affairs of the Republic of China under Contract No. MOEA 94-EC-17-A-08-S1-0006.

\section{Appendix}

The components of Eq. (2) are

$$
\begin{aligned}
& L_{x x}=\frac{\partial}{\partial x}\left(C_{11} \frac{\partial}{\partial x}\right)+\frac{\partial}{\partial y}\left(C_{44} \frac{\partial}{\partial y}\right)-C_{44} k_{z}^{2}, \\
& L_{x y}=\frac{\partial}{\partial x}\left(C_{12} \frac{\partial}{\partial y}\right)+\frac{\partial}{\partial y}\left(C_{44} \frac{\partial}{\partial x}\right), \\
& L_{x z}=i k_{z}\left(\frac{\partial}{\partial x} C_{12}+C_{44} \frac{\partial}{\partial x}\right) \\
& L_{y x}=\frac{\partial}{\partial y}\left(C_{12} \frac{\partial}{\partial x}\right)+\frac{\partial}{\partial x}\left(C_{44} \frac{\partial}{\partial y}\right), \\
& L_{y y}=\frac{\partial}{\partial x}\left(C_{44} \frac{\partial}{\partial x}\right)+\frac{\partial}{\partial y}\left(C_{11} \frac{\partial}{\partial y}\right)-C_{44} k_{z}^{2}, \\
& L_{y z}=i k_{z}\left(\frac{\partial}{\partial y} C_{12}+C_{44} \frac{\partial}{\partial y}\right), \\
& L_{z x}=i k_{z}\left(C_{12} \frac{\partial}{\partial x}+\frac{\partial}{\partial x} C_{44}\right), \\
& L_{z y}=i k_{z}\left(C_{12} \frac{\partial}{\partial y}+\frac{\partial}{\partial y} C_{44}\right) \\
& L_{z z}=\frac{\partial}{\partial x}\left(C_{44} \frac{\partial}{\partial x}\right)+\frac{\partial}{\partial y}\left(C_{44} \frac{\partial}{\partial y}\right)-C_{11} k_{z}^{2} .
\end{aligned}
$$


*Electronic address: yinghung@gate.sinica.edu.tw

$\dagger$ Electronic address: mechang@gate.sinica.edu.tw

${ }^{1}$ Y. Tanaka, Y. Tomoyasu, and Shin-ichiro Tamura, Phys. Rev. B 62, 7387 (2000).

${ }^{2}$ J. O. Vasseur, P. A. Deymier, B. Chenni, B. Djafari-Rouhani, L. Dobrzynski, and D. Prevost, Phys. Rev. Lett. 86, 3012 (2001).

${ }^{3}$ S. Yang, J. H. Page, Z. Liu, M. L. Cowan, C. T. Chan, and Ping Sheng, Phys. Rev. Lett. 93, 024301 (2004).

${ }^{4}$ M. Wilm, A. Khelif, S. Ballandras, V. Laude, and B. DjafariRouhani, Phys. Rev. E 67, 065602(R) (2003).

${ }^{5}$ A. Khelif, M. Wilm, V. Laude, S. Ballandras, and B. DjafariRouhani, Phys. Rev. E 69, 067601 (2004).

${ }^{6}$ J. Mei, Z. Y. Liu, and C. Qiu, J. Phys.: Condens. Matter 17, 3735 (2005).

${ }^{7}$ Z. Liu, X. Zhang, Y. Mao, Y. Y. Zhu, Z. Yang, C. T. Chan, and P. Sheng, Science 289, 1734 (2000).

${ }^{8}$ M. M. Sigalas and N. Garcia, J. Appl. Phys. 87, 3122 (2000).

${ }^{9} \mathrm{Ph}$. Lambin, A. Khelif, J. O. Vasseur, L. Dobrzynski, and B. Djafari-Rouhani, Phys. Rev. E 63, 066605 (2001)
${ }^{10}$ M. Kafesaki and E. N. Economou, Phys. Rev. B 60, 11993 (1999).

${ }^{11}$ C. Goffaux and José Sánchez-Dehesa, Phys. Rev. B 67, 144301 (2003).

${ }^{12}$ R. L. Chern, C. C. Chang, C. C. Chang, and R. R. Hwang, Phys. Rev. E 68, 026704 (2003).

${ }^{13}$ C. C. Chang, J. Y. Chi, R. L. Chern, C. C. Chang, C. H. Lin, and C. O. Chang, Phys. Rev. B 70, 075108 (2004).

${ }^{14}$ D. M. Sullivan, Electromagnetic Simulation Using The FDTD Method (IEEE Press, New York, 2000).

${ }^{15}$ J. O. Vasseur, B. Djafari-Rouhani, L. Dobrzynski, M. S. Kushwaha, and P. Halevi, J. Phys.: Condens. Matter 6, 8759 (1994).

${ }^{16}$ G. Wang, J. Wen, Y. Liu, and X. Wen, Phys. Rev. B 69, 184302 (2004).

${ }^{17}$ C. C. Mei, J-L. Auriault, and C. O. Ng, Some Applications of the Homogenization Theory, Advances in Applied Mechanics, Vol. 32, edited by J. Hutchinson and T. Y. Wu (Academic Press, New York, 1996). 